\title{
Greater trochanteric bone flap grafting with vascular pedicles for the treatment of femoral head osteonecrosis in patients with systemic lupus erythematosus: A retrospective study
}

Qiang Yang ( $\square$ yangqiang2020@yeah.net)

Nanyang orthopedic hospital

Jixue Zhou

Nanyang orthopedic hospital

Lei Li

Nanyang orthopedic hospital

Zhaopeng Guo

Nanyang orthopedic hospital

Xiaolei Tian

Nanyang orthopedic hospital

\section{Research article}

Keywords: Femoral head osteonecrosis, Systemic lupus erythematosus, greater trochanteric bone flap, vascular pedicles

Posted Date: May 26th, 2020

DOI: https://doi.org/10.21203/rs.3.rs-30668/v1

License: (c) (i) This work is licensed under a Creative Commons Attribution 4.0 International License.

Read Full License 


\section{Abstract}

Background: Osteonecrosis of the femoral head is a complication of systemic lupus erythematosus,that affect the patient's quality of life seriously. This article reviewed the radiographs of osteonecrosis of the femoral head in patients with systemic lupus erythematosus and assessed the effect of using the greater trochanteric bone flap grafting with vascular pedicles.

Methods: We retrospectively reviewed 17 patients (26 hips) with systemic lupus erythematosus who underwent the greater trochanteric bone flap grafting with vascular pedicles for the treatment of osteonecrosis of the femoral head. According to Ficat and Arlet classification, 16 hips were in stage II; 10 hips were in stage III. All patients were followed up for a mean of 32 months (ranging 12 48 months) and were assessed clinically and radiologically according to Harris scoring.

Results: No hips were treated with total hip arthroplasty in the follow-up. The mean HHS was improved from preoperative 69.1 points (ranging 52-83 points) to postoperative 89.4 points (ranging $56-100$ points). At the latest follow-up, of 26 hips, radiographically 21 hips (80.77\%) were improved, 3 hips (11.54\%) were unchanged and just 2 hips (7.69\%) were worse.

Conclusion: The greater trochanteric bone flap grafting with vascular pedicles was successful in maintaining joint function and in delaying the need for joint replacement procedure.

\section{Introduction}

Systemic lupus erythematosus (SLE) is a diffuse connective tissue disease with multiple system damage mediated by autoimmune reactions. Osteonecrosis of the femoral head (ONFH) is one of the most serious complications of $\operatorname{SLE}(1)$. Its morbidity has been reported to be about $4 \%-30 \%$ with an overall average of $10 \%$, which is higher than that of general populatio(1-3). The etiology of this disorder has not been clarified, and no prophylaxis has been established to date. Although the pathogenesis remains unclear, involvement of high-dose corticosteroids therapy(4), immunosuppressive drug therapy(5), hypercoagulability(6), and lipid metabolism abnormality(7) has been suggested. ONFH seriously affects the quality of life of patients, which is an urgent medical problem to be solved.

Total hip arthroplasty (THA) is an effective method to treat ONFH(8), which can reduce patients' pain and improve the function of hip joint. Without early surgical intervention, total hip advanced lesions would appear, and eventually THA was unavoidable. However, SLE is more common in younger patients, premature THA will face many problems, such as prosthetic loosening, infection and repeated revision surgery $(9,10)$. In addition, patients with SLE who take hormones for a long time often have other systemic diseases, which increase the risk of surgery and perioperative complications(9). These will have serious impact on the patient's physiology, psychology and economy, so total hip arthroplasty for younger patient populations need be paid very careful attention. Therefore, on the basis of improvement in symptoms, what kind of surgical methods to delay or even avoid artificial joint replacement has become the focus in theapy research of ONFH. 
Current research methods include core decompression surgery, osteotomy and vascularized bone graft, the therapeutic effect of core decompression and osteotomy has been questioned for the patients whose cartilage damaged and collapsed $(11,12)$. It had reported that vascularized greater trochanter bone flap transplantation was effective for the treatment of $\mathrm{ONFH}$, and the femoral head preservation rate was $88.2 \%(13)$. At present, there is few studies have reported the treatment of ONFH with vascularized bone flap transplantation. At present, few studies have reported vascularized bone flap graft for the treatment of SLE complicated with ONFH. The purpose of this study was to investigate the effect of the vascularized bone graft for the treatment of secondary ONFH caused by SLE.

\section{Materials And Methods}

All procedures were approved by an Institutional Ethics Review Committee of Nanyang Orthopaedic Hospital. Informed consent was obtained from all individuals who participated in this study.

\section{Patients}

Since July 2012 to June 2016, 17 patients (26 hips) with ONFH underwent the procedure of the trochanteric bone flap pedicles grafting. There were 4 males and 13 females. The age of the patients ranged from 18 to 42 years (mean 41.1 years ), cause ranged from 1 6 years and the average duration were 3.4 years. All patients had received corticosteroids threatment.

\section{ONFH diagnosis and classification}

All the patients were corresponding to SLE diagnosis standards established by the 1982 American Rheumatology Association criteria(14). ONFH was identified by one or more of the following imaging techniques: plain X-ray, computsd tomography and MRI. According to the Ficat and Arlet classification(15), 16 hips were in stage II (61.5\%); 10 hips were in stage III (38.5\%). The diagnosis of osteonecrosis was confirmed in all cases by a histological examination of the subchondral bone that was obtained from a core biopsy of the femoral head during the surgery.

\section{Surgical procedure}

After the patient is anesthetized, they were placed in the supine position with the ilium elevated to $60^{\circ}$. A skin incision was made $4 \mathrm{~cm}$ distal to the iliac crest down to the top of greater trochanter, and then extend vertically down along the anterior margin of the trochanter. After we identifing the transverse branch of the lateral femoral circumflex vessel, we separated these vessels to where they entered the greater trochanter. We harvested a vascular graft bone flap about $3 \times 2 \mathrm{~cm}$ from the anterolateral side of the greater trochanter. Then, we harvested a volume of $1-2 \mathrm{~cm}^{3}$ of cancellous bone from the greater trochanter area.The hip capsule was incised in a $T$ shape to expose the femoral head and neck. We made an approximately $2 \times 2 \mathrm{~cm}$ bone window at the femoral head-neck junction using an osteotome. According to the imaging evaluation and the judgment of the surgeon,we determined the range of femoral head necrosis.We used the high-speed drill and curette spatula to remove the necrotic bone tissue in the 
femoral head, until the necrotic tissue is removed compeletly and fresh blood flows out. The vascularized bone flap was implanted into the femoral head lesion area after the necrotic bone was removed, and the cancellous bone was filled in insufficient places. The bone flap and the filled cancellous bone were properly tamped to restore the shape of the collapsed femoral head.We required bleeding from the cancellous surface of the greater trochanter graft as an indication of vessel patency.

\section{Clinical evaluation}

Clinical follow-up is performed every 3 months for 1 year and annually thereafter. The mean length of follow-up was 32 months (ranging 12 to 48 months) in our hospital. Before the procedure and at each follow-up, patients were evaluated using the Harris Hip Score (HHS)(16).The HHS is an objective index of hip joint function that quantifies the four categories of (1) pain, (2) walking function and activities of daily living, (3) extent of deformity and (4) joint range of motion, with higher values indicating greater functionality. Harris score no less than 80 points is defined as clinical success. Clinical outcomes were categorised by HHS results at the most recent follow-up as follows: excellent, 90-100; good, 80-89; fair, 70-79; or poor, less than 70.

\section{Radiographic evaluation}

Radiographic progressions of the femoral head collapse (pre-surgery to the most recent follow-up) were evaluated in hips of each classification and staging. According the final radiographs, we are divided into three categories:(1) Improved-those cases in which the osteonecrosis had healed or was being replaced

with new bone formation. For the Stage II lesion, the density of cystic lesion had increased with trabecular formation of the greater trochanteric bone flap. For the Stage III lesion, the collapsed lesion healed or became more rounded with trabecular formation of the tip of the greater trochanteric bone flap.

(2)Remained unchanged, and the preoperative imaging data showed no significant difference.(3) Worsestaging of disease progression,collapse further aggravated.

\section{Results}

\section{Clinical Results}

The Surgical procedure took 50 to 90 minutes to complete with the mean of 65 minutes.Mean perioperative blood loss was $320 \mathrm{~mL}$ (range $220-510 \mathrm{~mL}$ ).All patients received perioperative prophylactic antibiotics until after removal of the drains. There were no intraoperative complications. Fat necrosis of incision occurred in one patient postoperatively and the incision healed untill 3 weeks after debridement. There were no other complications, such as infection, hematoma and deep vascular thrombosis, during the period of follow-up.

\section{Harris hip scores}

At last follow-up,The mean HHS was improved from preoperative 69.1 points (ranging 52-83 points) to postoperative 89.4 points (ranging $56-100$ points) (The overall average increase in HHSs is shown in 
Figure 1).The mean HHS was improved from preoperative 78.4 points (ranging $74-83$ points) to postoperative 95.4 points (ranging 79-100 points) for femoral heads with stage II disease and from preoperative 59.8 points (range 52-68 points) to postoperative 83.4 points (range 56-94 points) for

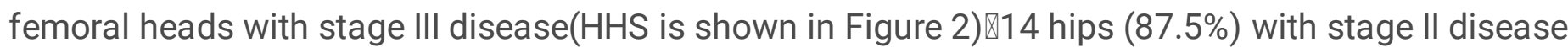
achieved excellent and good results and 8 of 10 hips (80\%)with stage III disease had excellent and good results (Table 1$)$.

\section{Radiographic outcomes}

All patients underwented radiological examination at follow-up. Of 26 hips, radiographically 21 hips (80.77\%) improved, 3 hips (11.54\%) were unchanged and just 2 hips (7.69\%) were worse. According to the staging, 14 hips of staging II (67.3\%) showed improvement, 2 hips of staging $I(12.5 \%)$ appeared unchanged and 1 hips of staging II (6.3\%) appeared worse.7 hips of staging III (70\%) showed improvement (Figs. 3A and 3B),1 hips of staging III (10\%) appeared unchanged and 1 hip of staging III (10\%) appeared worse.

\section{Discussion}

A satisfactory treatment for osteonecrosis of the femoral head in patients with SLE remains elusive.Without operative intervention ONFH could result in collapse and deterioration of the joint. Some scholars reported(17) a 53\% rate of progression to collapse of the femoral head articular surface in 79 hips treated nonoperatively.Current treatment options include core decompression, various osteotomy techniques and vascularized bone graft(18-21).

Core decompression provides pain relief,especially for patients with earlier stages of avascular necrosis(22).There is evidence that core decompression is an effective intervention in lupus patients with Ficat stage I and II.Several studies report, failure of core decompression and progression of osteonecrosis in lupus patients, especially in patients with Ficat stage III(23). Maniwa(24) have evaluated the results of core decompression for osteonecrosis of the hip in patients with SLE. The report suggest that patients with avascular necrosis of the femoral head and lupus do not respond as well as nolupus patiens treated with core decompression.

In 1978, Sugioka(25) first introduced rotary osteotomy through greater trochanter of femur to treat ONFH区 which was intertrochanteric osteotomy of the femoral head necrosis rotation. The principle is the upper part of the front of the femoral head necrosis is transferred to the non-load-bearing parts, in order to prevent progressive collapse of the articular surface of the femoral head, and correct head and acetabulum mismatch caused by subluxation occured after the collapse of the articular surface of the femoral head. Then a variety of improved surgical procedures used in clinical, there is a big difference in the reported results. Although it is considered this kind of surgical mothod can prevent the collapse of the new weight-bearing area, but increased the incidence of instability and the corresponding joint osteoarthritis. Due to the high incidence of this surgical complication and followed by deformity of the proximal femur, the clinical application is limited. Greater trochanter of femur mainly comprised by 
spongy bone, have rich blood supply, bone flap have large range blood supply, the blood supply in the affected areas can be improved effectively after transplantation, the rebuilding process of the blood supply can be shortened.

The use of the free fibular flap for the treatment of ONFH was initiated in an effort to enhance revascularization and arrest the progression of the necrosis.Soucacos(26) reported using free vascularized fibular grafting for osteonecrosis of the femoral head in 184 hips, a mean follow-up of 11 years, only $7.6 \%$ of patients requiring total hip arthroplasty, and $62 \%$ patients without progression. However, the procedure of free fibular flap transfer is technical demanding and time consuming.Moreover, complications associated with the use of fibular flap, such as donor-site pain, peroneal nerve neuropathy and fracture of the femoral neck, have also been reported $(27,28)$.

The greater trochanter bony structure with cancellous bone, peripheral rich blood supply, transplantation can effectively improve the blood supply, reduce blood supply reconstruction process. The thin cortical bone is very similar to head of femur, is ideal selected area to treat ONFH; and is able to improve the mechanical properties of necrosis of the femoral head effectively. After removing the necrotic bone completely, the transferred greater trochanter and the remaining part of femoral metastases fit closely to promote fracture healing process; greater trochanter and femoral trabecular bone trabecular have same nature, which restored the continuity of cancellous bone trabecular, rebuilding the supporting role of trabecular bone of the femoral head, to recovey femoral neck bearing bracket, can be adapted to load normal joint activities; meanwhile as the cystic degeneration repair and new bone reconstruct, the femoral head restoring the force area, so that the stress beared by unit trabecular bone area reduced, the mechanical properties of the femoral head strengthened, preventing the collapse effectively. In addition, the vascularized bone flap can reconstruct a new blood supply system, accelerate the repair and rebuilding of bone necrosis, thereby restoring the biological characteristics of the femoral head necrosis. And by mechanics, we proved the cut of the greater trochanter does not damage the mechanical properties and structure of the proximal femur trochanter major stress conductive zones, thus from a mechanical point of view, the selection of bone flap from the greater trochanter is safe and feasible. The bone flap transplantation located in front of the femoral neck, this area is a region of low stress distribution, can reduce the impact on the mechanical characteristics of the proximal femur. This provides bone flap entering the channel and select specific operative incision from femur head and neck in greater trochanter vascularized bone grafting a strong theoretical basis, but also proves the safety of this surgical technique, and fewer complications.

On the use of vascularized the greater trochanteric bone flap graft treating 26 hips with secondary ONFH caused by SLE, by an average of 32 months of follow-up,medium-term clinical results were satisfactory,the excellent and good rate was $88.5 \%$, this surgery can slow down or even stop the progress of ONFH. During follow-up, femoral head necrosis increasing with the progression of stage in two patients, DSA angiography showed: blood filling poorly in vascularized bone or no contrast agent filling the femoral head, maybe caused by the twist or spasm of the vascular pedicle, or constriction from 
surrounding tissue swelling, until to the last follow-up, both patients have no other further treatment requirements in addition to functional rehabilitation treatment.

But we are also aware of some limitations of our study. First, we had no control group treated with alternative joint-preserving procedures. Second, the length of follow-up is only 32 monthes. Longer term outcome analysis will be necessary to prove the longevity of the procedure. Third, the current study has only 26 hips; therefore, significant statistical conclusions are more difficult to make.

\section{Conclusion}

The effects of the greater trochanteric bone flap in patients with lupus on plain x-ray changes and HHSs demonstrate the benefits of this technique. The greater trochanteric graft flap with pedicles are easy to perform and could be indicated in young patients with Ficat and Arlet stage II to III disease. Its could provide the necrotic femoral head with sufficient blood supply and prevention of femoral head collapse.

\section{Abbreviations}

SLE, systemic lupus erythematosus; ONFH ,Osteonecrosis of the femoral head; THA ,Total hip arthroplasty

\section{Declarations}

\section{Ethics approval and consent to participate}

We declar that all procedures were approved by an Institutional Ethics Review Committee of Nanyang Orthopaedic Hospital, and obtained the consent of the participants.

\section{Consent for publication}

All the contents obtained the consent of the patients for publication.

\section{Availability of data and materials}

All data generated or analysed during this study are included in this published article.

\section{Competing interests}

The authors declare that they have no competing interests.

\section{Fundings}

None.

\section{Authors' contributions}


Conception and design of the research:Qiang Yang; Acquisition, analysis and interpretation of data: Jixue Zhou and Lei Li; Statistical analysis: Zhaopeng Guo and Xiaolei Tian; Drafting the manuscript: Qiang Yang; Manuscript revision for important intellectual content: Qiang Yang. All authors have read and approved the manuscript.

\section{Acknowledgement}

Not applicable.

\section{References}

1. Gladman D, Dhillon N, Su J, Urowitz M. Osteonecrosis in SLE: prevalence, patterns, outcomes and predictors. Lupus. 2018;27(1):76-81.

2. Mertelsmann-Voss C, Lyman S, Pan TJ, Goodman S, Mandl LA. Arthroplasty Rates Are Increased Among US Patients with Systemic Lupus Erythematosus: 1991-2005. Journal of Rheumatology. 2014;41(5):867-74.

3. Kasturi S, Goodman S. Current Perspectives on Arthroplasty in Systemic Lupus Erythematosus: Rates, Outcomes, and Adverse Events. Current Rheumatology Reports. 2016;18(9):59.

4. Nevskaya T, Gamble MP, Pope JE. A meta-analysis of avascular necrosis in systemic lupus erythematosus: prevalence and risk factors. Clinical and Experimental Rheumatology. 2017;35(4):700.

5. Systemic lupus erythematosus in a multiethnic US cohort (LUMINA): XXIV. Cytotoxic treatment is an additional risk factor for the development of symptomatic osteonecrosis in lupus patients: results of a nested matched case-control study. Annals of the Rheumatic Diseases. 2006.

6. Oinuma K, Harada Y, Nawata Y, Takabayashi K, Abe I, Kamikawa K, et al. Sustained hemostatic abnormality in patients with steroid-induced osteonecrosis in the early period after high-dose corticosteroid therapy. Journal of Orthopaedic Science Official Journal of the Japanese Orthopaedic Association. 2000;5(4):374-9.

7. Belmont $H$, Lydon E. Avascular necrosis prevention with lipitor in lupus erythematosus. Lupus. 2005;14(10):869-70.

8. Li Z, Du Y, Xiang S, Feng B, Weng X. Risk factors of perioperative complications and transfusion following total hip arthroplasty in systemic lupus erythematosus patients. Lupus. 2019;28(9):096120331986260.

9. Kennedy JW, Wasim K. Total Hip Arthroplasty in Systemic Lupus Erythematosus: A Systematic Review. International Journal of Rheumatology. 2015;2015:1-8.

10. Zangger P, Gladman DD, Urowitz MB, Bogoch ER. Outcome of total hip replacement for avascular necrosis in systemic lupus erythematosus. Journal of Rheumatology. 2000;27(4):919.

11. Scully SP, Aaron RK, Urbaniak JR. Survival Analysis of Hips Treated with Core Decompression or Vascularized Fibular Grafting Because of Avascular Necrosis. Journal of Bone and Joint Surgery, 
American Volume. 1998;80(9):1270-5.

12. Halland AM, Klemp P, Botes D, Van Heerden B, Loxton A, Scher AT. AVASCULAR NECROSIS OF THE HIP IN SYSTEMIC LUPUS ERYTHEMATOSUS: THE ROLE OF MAGNETIC RESONANCE IMAGING. Rheumatology. 1993;32(11):972-6.

13. Zhao D, Wang B, Guo L, Yang L, Tian F. Will a Vascularized Greater Trochanter Graft Preserve the Necrotic Femoral Head? Clinical Orthopaedics \& Related Research. 2010;468(5):1316-24.

14. Tan EM, Cohen AS, Fries JF, Masi AT, Winchester RJ. The 1982 revised criteria for the classification of systemic lupus erythematosus. . Arthritis \& Rheumatology. 1982;25(11):1271-7.

15. J FRA. Necrosis of the femoral head. In: Hungerford DS,editor Ischemia and Necrosis of Bone Baltimore, MD: Williams \&Wilkins;. 1980:55-74.

16. Harris WH. Traumatic arthritis of the hip after dislocation and acetabular fractures: treatment by mold arthroplasty. An end-result study using a new method of result evaluation. The Journal of bone and joint surgery American volume. 1969;51(4):737-55.

17. OHZONO K, SAITO M, SUGANO N, TAKAOKA K, ONO K. The Fate of Nontraumatic Avascular Necrosis of the Femoral Head: A Radiologic Classification to Formulate Prognosis. Clin Orthop Relat Res. 1992;277(277):73-8.

18. Pedesen DR, Brown TD, Poggie RA. Finite element characterization of a porous tantalum material for treatment of avascular necrosis. Trans Orthop Res Soc. 1997;22:598.

19. Shuler MS, Rooks MD, Roberson JR. Porous Tantalum Implant in Early Osteonecrosis of the Hip: Preliminary Report on Operative, Survival, and Outcomes Results. Journal of Arthroplasty. 2007;22(1):0-31.

20. Tsao, A. K. Biomechanical and Clinical Evaluations of a Porous Tantalum Implant for the Treatment of Early-Stage Osteonecrosis. Journal of Bone \& Joint Surgery American Volume. 2005;87(suppl_2):22.

21. Veillette, J.H. C. Survivorship Analysis and Radiographic Outcome Following Tantalum Rod Insertion for Osteonecrosis of the Femoral Head. Journal of Bone \& Joint Surgery. 2006;88(suppl_3):48.

22. Miyahara HdS, Rosa BB, Hirata FY, Gurgel HdMC, Ejnisman L, Vicente JRN. What is the role of core decompression in the early stages of osteonecrosis of the femoral head? Evaluation of the surgical result by functional score and radiological follow-up. Revista Brasileira De Ortopedia. 2018;53(5):537-42.

23. MONT, Michael A, FAIRBANK, Adrian C, MICHELLE, HUNGERFORD, et al. Core Decompression for Osteonecrosis of the Femoral Head in Systemic Lupus Erythematosus. Clinical Orthopaedics \& Related Research. 1997;334(334):91-7.

24. Maniwa S, Nishikori T, Furukawa S, Kajitani K, Iwata A, Nishikawa U, et al. Evaluation of core decompression for early osteonecrosis of the femoral head. Archives of Orthopaedic \& Trauma Surgery. 2000;120(5-6):241-4.

25. Sugioka Y. Transtrochanteric anterior rotational osteotomy of the femoral head in the treatment of osteonecrosis of the femoral head in the treatment of osteonecrosis affecting the hips; a new 
osteotomy operation. Clin Orthop. 1978;130.

26. Soucacos PN, Beris AE, Malizos K, Koropilias A, Zalavras H, Dailiana Z. Treatment of Avascular Necrosis of the Femoral Head With Vascularized Fibular Transplant. Clinorthoprelatres. 2001;386:120-30.

27. URBANIAK JR. Treatment of osteonecrosis of the femoral head with free vascularized fibular grafting. : A long-term follow-up study of one hundred and three hips. J Bone Joint Surg. 1995;77.

28. Urbaniak JR, Harvey EJ. Revascularization of the Femoral Head in Osteonecrosis. J Am Acad Orthop Surg. 1998;6(1):44-54.

\section{Table}

Table 1. Clinical Outcome of HSS in osteonecrosis of the femoral head.

Ficat and Alert Stages

Stage II Stage III

\begin{tabular}{lccc} 
Outcome & \multicolumn{2}{c}{ Case } & \multicolumn{2}{c}{6} \\
Excellent & 19 & 13 & 2 \\
Good & 3 & 1 & 1 \\
Fair & 3 & 2 & 1 \\
Poor & 1 & 0 & 10 \\
Total & 26 & 16 &
\end{tabular}

\section{Figures}




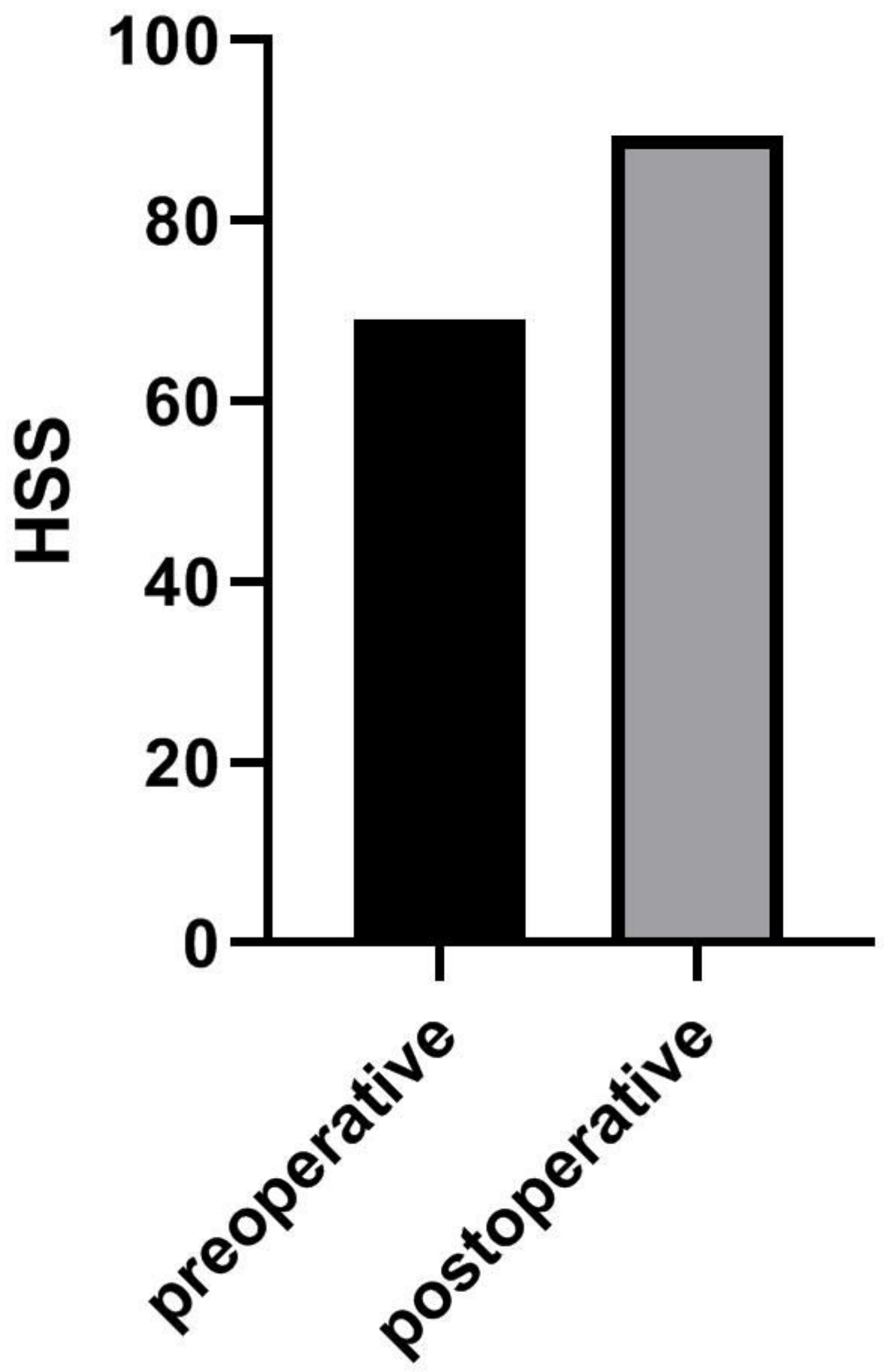

Figure 1

The effect of the greater trochanteric bone flap with vascular pedicles grafting on HHS is shown. 


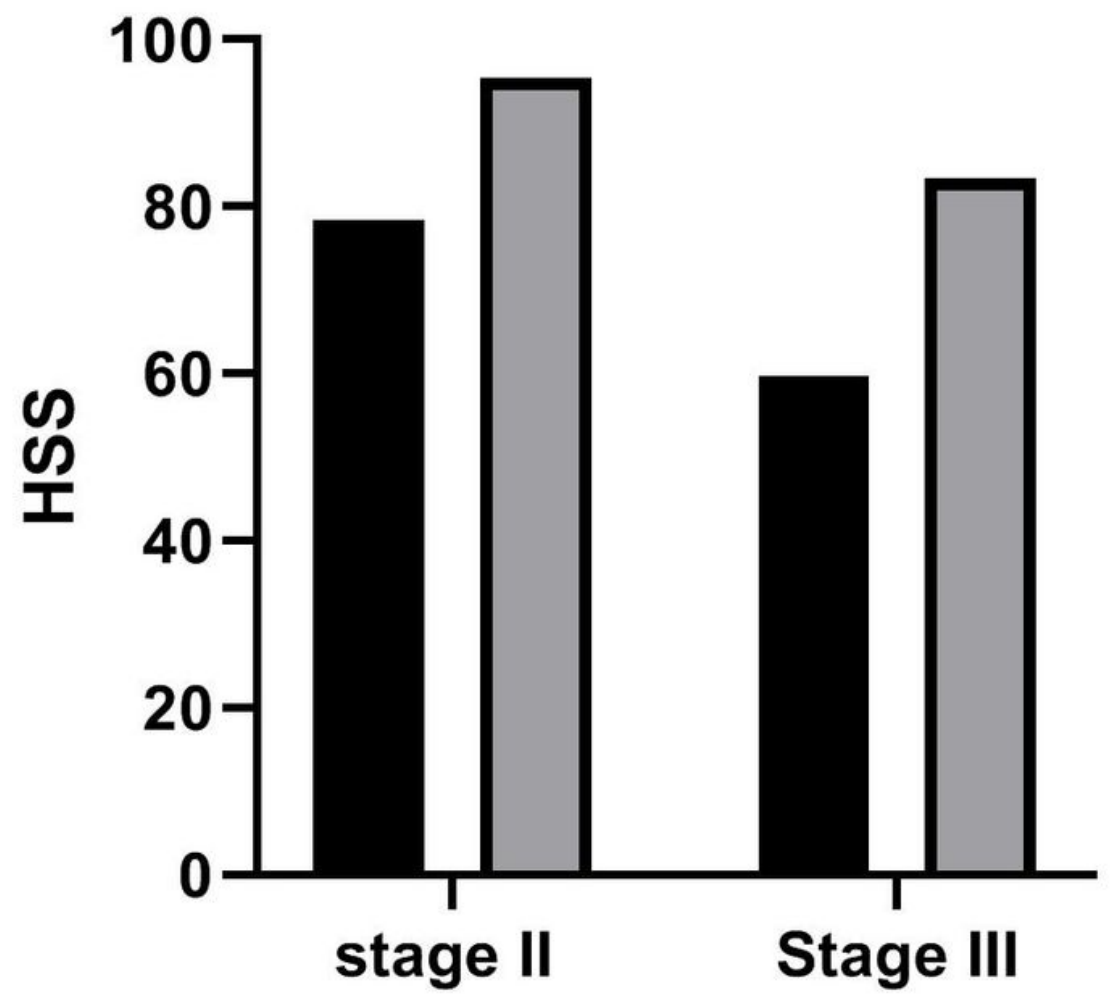

m preoperative

$\square$ postoperative

Figure 2

The effect of the greater trochanteric bone flap with vascular pedicles grafting on Harris hip score (HHS) in stage II and III is shown.

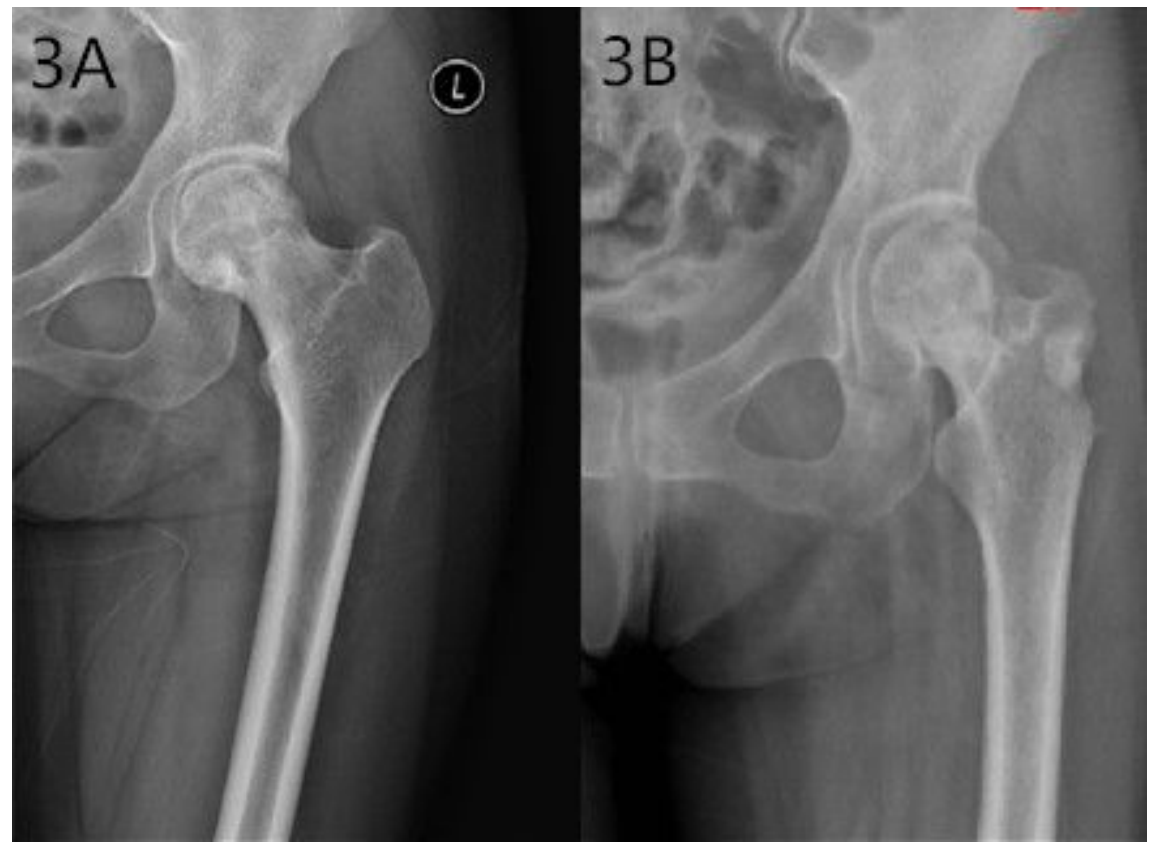

Figure 3 
A 32-year-old woman with lupus and stage III osteonecrosis of the left femoral head with collapse in the preoperative X-ray examination. Anteroposterior radiographs were taken preoperatively (3A) and 36 months postoperatively (3B), at which time the greater trochanter bone flap graft was well incorporated and the forma of the femoral head did not show progressive collapse. 